\title{
Improved Crank-Nicolson method for modelling diffusion of electromagnetic fields in hysteretic soft magnetic laminations.
}

\author{
Tanja Van Hecke \\ Faculty of Engineering and Architecture, Ghent University, \\ V. Vaerwyckweg 1, 9000 Ghent (Belgium), \\ E-mail: Tanja.VanHecke@ugent.be
}

\begin{abstract}
This paper studies the use of time stepping methods to integrate the equations which model the magnetodynamic fields in ferromagnetic laminations. A new high-order time stepping method is compared to well-known time stepping methods (Euler, Crank Nicolson, ...) for a diffusion problem of electromagnetic fields in magnetic laminations with hysteresis. For validation purposes, firstly a theoretical problem with analytical solution is solved. Afterwards, more realistic problems are studied. As the presented method is $A$-stable, accurate numerical loops can be computed, even in the case of hysteresis effects.
\end{abstract}

Keywords: Crank-Nicolson, Lamination, Nonlinear diffusion, Timestepping

\section{Introduction}

In the field of modern electromagnetics the study of nonlinear diffusion of electromagnetic fields in conducting media with gradual magnetic transitions is an important problem. In material exhibiting hysteresis, the magnetic induction $B$ is not only a function of the magnetic field $H$, but also of its history. When simulating electromagnetic systems such as transformers and electrical machines, it is useful to implement in the numerical model the magnetic material behaviour in a detailed way, especially if one of the goals is to determine the losses in the magnetic laminations subject to dynamic fields. A model for diffusion of fields in a lamination exists [1] and predicts the average induction in the lamination as a function of the magnetic field waveform at the edge, based on measured hysteresis loops in strips of the material. If the field waveform is considered in a sufficient number of points of e.g. a finite element model of an electromagnetic system, the lamination model can be used to estimate the losses in the lamination in a realistic way. Many studies focus on improving the integration in time of the parabolic partial differential equations behind this problem. We compare the performance of existing numerical methods with a method where a higher order method of lines is used.

The paper first explains a simple electromagnetic diffusion problem of magnetic fields in a lamination with nonlinear hysteretic properties (section 2). Then, it gives an overview of methods for space discretization (section 3) and time stepping, presents an improved version of the Crank-Nicolson method (section 4), and proves its stability (section 5). For validation purposes, all presented methods are in section 6 applied to the simple electromagnetic diffusion problem of magnetic fields of section 2, for which an exact analytical solution is available. The lamination model in section 7 is a similar diffusion problem as in section 2 , but applicable to all magnetic materials. Several algorithm related parameters are investigated in order to accurately reproduce the dynamic hysteresis loops in the material.

\section{Model of diffusion of electromagnetic fields}

We consider the problem of nonlinear diffusion of electromagnetic fields in conducting media with gradual magnetic transitions. Therefore an Euclidean coordinate system is defined with the $x$-axis perpendicular to the lamination. Throughout the lamination, the time periodic unidirectional flux $\phi(t)$ flows in the $z$-direction and the magnetic field $\mathbf{H}$ has only one component: $\mathbf{H}=H(x, t) \mathbf{1}_{z}$. Neglecting the edge effects, we may assume the electric fields $\mathbf{E}$ and the flux density $\mathbf{B}$ to vary in the $x$-direction only. They can be denoted by their only component that differs from zero: $\mathbf{E}=E(x, t) \mathbf{1}_{y}$ and $\mathbf{B}=B(x, t) \mathbf{1}_{z}$. 
Maxwell's equations become

$$
\begin{aligned}
& \frac{\partial H}{\partial x}=-\sigma E \\
& \frac{\partial E}{\partial x}=-\frac{\partial B}{\partial t} .
\end{aligned}
$$

The particular diffusion model (3) is described in [2], where $n$ is a measure of the sharpness of magnetic transition and $k$ is the proportionality factor of $h$ and $b^{n}$ as in (4).

$$
\left\{\begin{array}{l}
\frac{\partial^{2} b^{n}}{\partial x^{2}}=k \sigma \frac{\partial b}{\partial t} \\
b(x, 0)=0 \\
b(0, t)=H_{0} t^{\frac{1}{n-1}}
\end{array}\right.
$$

$H_{0}$ determines the magnetic field at the border $(x=0)$. The shifted magnetic field $h$ and magnetic flux densisty $b$ are defined by

$$
h=H-H_{c}, \quad b=B+B_{c} \quad \text { and } \quad h=\frac{b^{n}}{k},
$$

with $H_{c}$ the value for the initial magnetic field $H$ and $-B_{c}$ the value for the initial magnetic flux density $B$. The electric conductivity $\sigma$ gives rise to eddy currents in the lamination if changes in flux density occur. As an equivalent formulation of (3) is

$$
\left\{\begin{array}{l}
\frac{\partial^{2} H}{\partial x^{2}}=\frac{\sigma k^{\frac{1}{n}}}{n}\left(H-H_{c}\right)^{\frac{1}{n}-1} \frac{\partial H}{\partial t} \\
H(x, 0)=H_{c} \\
H(0, t)=H_{c}+\frac{1}{k}\left(H_{0} t^{\frac{1}{n-1}}\right)^{n}
\end{array}\right.
$$

Mayergoyz [2] proves that the exact solution is given by

$$
b(x, t)=H_{0} t^{\frac{1}{n-1}}\left(1-\frac{x}{v t}\right)^{\frac{1}{n-1}}
$$

with velocity $v=\sqrt{\frac{n}{(n-1)} \frac{1}{k \sigma}} H_{0}^{\frac{n-1}{2}}$.

The analytical - and thus exact - solution of this diffusion problem will be used in section 6 to validate the numerical methods, in particular the improved Crank-Nicolson method.

\section{$3 \quad$ Method of lines}

To approach the differentiation $\frac{\partial^{2} H}{\partial x^{2}}$ the technique of finite elements can be used [3], but we choose to use the method of lines [4] based on finite differences. The well-known Crank-Nicolson method (21) [5] is based on the approximation

$$
M_{2}=\frac{1}{(\Delta x)^{2}}\left(H_{j}^{i+1}-2 H_{j}^{i}+H_{j}^{i-1}\right)
$$

of $\frac{\partial^{2} H}{\partial x^{2}}$, with $H_{j}^{i}=H\left(x_{i}, t_{j}\right)$. This difference form transforms the partial differential equation $(5)$ into a ordinary differential equation of the form

$$
\frac{d H}{d t}=f(H, t)
$$

Note that $\frac{\partial^{2} H}{\partial x^{2}}=M_{2}+\mathbf{O}(\Delta x)^{2}$.

A better approximation $\frac{\partial^{2} H}{\partial x^{2}}=M_{4}+\mathbf{O}(\Delta x)^{4}$ can be made with

$$
M_{4}=\frac{1}{(\Delta x)^{2}}\left(-\frac{1}{12} H_{j}^{i+2}+\frac{4}{3} H_{j}^{i+1}-\frac{5}{2} H_{j}^{i}+\frac{4}{3} H_{j}^{i-1}-\frac{1}{12} H_{j}^{i-2}\right) .
$$




\section{Timestepping methods}

In this paragraph, we give an overview of well known timestepping methods, and we explain the improved Crank-Nicolson method.

Several families of methods have been developed to solve the first order problem

$\frac{d u}{d t}=f(u, t)$ based on two types of methods: the multistep methods and the Runge-Kutta methods [6] [7]. The difference can be found in the information that is used to find $H_{j+1}$ out of $H_{j}$ : multistep methods

$$
u_{j+1}=\sum_{i=0}^{p} a_{i} u_{j-i}+\Delta t \sum_{i=0}^{p} b_{i} f_{j-i}+\Delta t b_{-1} f_{j+1}
$$

use information at previous time steps, while Runge-Kutta methods

$$
\left\{\begin{array}{l}
u_{j+1}=u_{j}+\Delta t \sum_{l=1}^{s} b_{l} K_{l} \\
K_{l}=f\left(u_{j}+\Delta t \sum_{i=1}^{s} a_{l i} K_{i}, t_{j}+c_{l} \Delta t\right), \quad l=1,2, \ldots, s
\end{array}\right.
$$

use information at intermediary steps in time in $\left[t_{j}, t_{j+1}\right]$ as $0 \leq c_{i} \leq 1, i=1,2, \ldots, s$. Runge-Kutta methods can be compactly represented by the Butcher array [8]

$$
\begin{array}{c|c}
c & A \\
\hline & b^{T}
\end{array}
$$

with $A$ the $(s \times s)$-matrix with elements $a_{i j}$ and $b$ and $c$ the $(s \times 1)$-matrices with elements respectively $b_{i}$ and $c_{i}$.

Higher order methods require knowledge at intermediate points between $u_{j}$ and $u_{j+1}$. As this information is not always available, lower order methods are popular. Moreover in the case of partial differential equations, improvements of the accuracy of the time integration, will be lost by the errors of the second order discretization of $x$.

Multistep methods achieve a high order of accuracy by involving $u_{j}, u_{j-1}, u_{j-2}, \ldots, u_{j-p}$ for the determination of $u_{j+1}$. A special class of multistep methods is the family of backward difference formulae. The two-step, implicit, second order backward difference formula (BDF2)

$$
u_{j+1}=\frac{4}{3} u_{j}-\frac{1}{3} u_{j-1}+\frac{2}{3} \Delta t f_{j+1}
$$

The three-step, implicit, third order backward difference formula (BDF3)

$$
u_{j+1}=\frac{18}{11} u_{j}-\frac{9}{11} u_{j-1}+\frac{2}{11} u_{j-2}+\frac{6}{11} \Delta t f_{j+1}
$$

Special multistep methods are the first order explicit forward Euler method

$$
u_{j+1}=u_{j}+\Delta t f\left(u_{j}, t_{j}\right)
$$

and the first order implicit backward Euler method

$$
u_{j+1}=u_{j}+\Delta t f\left(u_{j+1}, t_{j+1}\right)
$$

The method (15) is also a special Runge-Kutta method with Butcher array

$$
\begin{array}{l|l}
0 & \\
\hline & 1
\end{array}
$$

while the method (16) is the first order BDF1 method.

Another special Runge-Kutta method is the second order explicit method of Heun

$$
\left\{\begin{array}{l}
u_{j+1}=u_{j}+\frac{\Delta t}{2}\left(f\left(u_{j}, t_{j}\right)+f\left(\hat{u}_{j+1}, t_{j+1}\right)\right) \\
\hat{u}_{j+1}=u_{j}+\Delta t f\left(u_{j}, t_{j}\right)
\end{array}\right.
$$

It can be seen as the forward Euler method as predictor and the trapezoidal rule as corrector and is also the explicit second order Runge-Kutta method

$$
\begin{array}{l|ll}
0 & & \\
1 & 1 & \\
\hline & \frac{1}{2} & \frac{1}{2}
\end{array} .
$$


The trapezoidal rule

$$
u_{j+1}=u_{j}+\frac{\Delta t}{2}\left(f\left(u_{j}, t_{j}\right)+f\left(u_{j+1}, t_{j+1}\right)\right)
$$

as the mean of the forward and the backward Euler method, is a second order multistep method and is popular due to its stability characteristics. The conventional Crank-Nicolson method is based on this trapezoidal rule with (7) as function evaluations, which leads to

$$
u_{j+1}^{i}=u_{j}^{i}+\frac{\Delta t}{2}\left(\frac{\left(u_{j+1}^{i+1}-2 u_{j+1}^{i}+u_{j+1}^{i-1}\right)+\left(u_{j}^{i+1}-2 u_{j}^{i}+u_{j}^{i-1}\right)}{(\Delta x)^{2}}\right) .
$$

When using (9) instead of (7) in combination with the trapezoidal rule, a method

$u_{j+1}^{i}=u_{j}^{i}+\frac{\Delta t}{2(\Delta x)^{2}}\left(\frac{4}{3}\left(u_{j}^{i+1}+u_{j+1}^{i+1}+u_{j}^{i-1}+u_{j+1}^{i-1}\right)-\frac{\left(u_{j}^{i+2}+u_{j+1}^{i+2}+u_{j}^{i-2}+u_{j+1}^{i-2}\right)}{12}-\frac{5}{2}\left(u_{j}^{i}+u_{j+1}^{i}\right)\right)$.

arises which can be seen as an improved version of the Crank-Nicolson method.

\section{Stability}

A method is called zero-stable if small perturbations of data yield bounded perturbations of the numerical solution when $\Delta t \rightarrow 0$. A better guarantee of stability is given by absolute stable methods. For a problem where the analytical solution tends to zero, they produce a numerical solution $\left(u_{n}\right)_{n>0}$ with $\lim _{n \rightarrow+\infty} u_{n}=0$. The forward Euler method is absolutely stable only for limited $\Delta t$-values, while the backward Euler method and the trapezoidal rule are unconditionally absolutely stable, also called $A$ stable. The Dahlquist barrier [8] limits the order of A-stable multistep methods to two. Explicit methods cannot be A-stable. This makes the forward Euler method and Heun's method less interesting although their implementation is more favourable.

The classical Crank-Nicolson method applied on the model problem $y^{\prime}=\lambda y$ ( $\lambda$ a positive real number and $y$ of dimension $m$ ) with an exact solution tending to zero as time tends to infinity, involves the solution of the system $A y_{n+1}=B y_{n}[9]$.

In case of $m=5: y_{n}=\left(u_{n}^{0}, u_{n}^{1}, u_{n}^{2}, u_{n}^{3}, u_{n}^{4}\right)^{T}$,

$$
A=\left(\begin{array}{cccccc}
1 & 0 & 0 & 0 & 0 & 0 \\
-\rho / 2 & 1+\rho & -\rho / 2 & 0 & 0 & 0 \\
0 & -\rho / 2 & 1+\rho & -\rho / 2 & 0 & 0 \\
0 & 0 & -\rho / 2 & 1+\rho & -\rho / 2 & 0 \\
0 & 0 & 0 & -\rho / 2 & 1+\rho & -\rho / 2 \\
0 & 0 & 0 & 0 & 0 & 1
\end{array}\right)
$$

with $\rho=\Delta t /(\Delta x)^{2}$ and

$$
B=\left(\begin{array}{cccccc}
1 & 0 & 0 & 0 & 0 & 0 \\
\rho / 2 & 1-\rho & \rho / 2 & 0 & 0 & 0 \\
0 & \rho / 2 & 1-\rho & \rho / 2 & 0 & 0 \\
0 & 0 & \rho / 2 & 1-\rho & \rho / 2 & 0 \\
0 & 0 & 0 & \rho / 2 & 1-\rho & \rho / 2 \\
0 & 0 & 0 & 0 & 0 & 1
\end{array}\right)
$$

The asymptotic behaviour is determined by the eigenvalues of the matrix $M=A^{-1} B$. As the requirement $\left|\lambda_{i}\right| \leq 1$ is naturally fulfilled, the conventional Crank-Nicolson method is unconditionally stable. Similarly the method (22) is unconditionally stable.

In case of $m=7\left(y_{n}=\left(u_{n}^{0}, u_{n}^{1}, u_{n}^{2}, u_{n}^{3}, u_{n}^{4}, u_{n}^{5}, u_{n}^{6}\right)^{T}\right)$, the matrices $A$ and $B$ related to method (22) can 
be written as respectively (25) and (26).

$$
A=\left(\begin{array}{ccccccc}
1 & 0 & 0 & 0 & 0 & 0 & 0 \\
0 & 1 & 0 & 0 & 0 & 0 & 0 \\
\rho / 12 & -4 \rho / 3 & 1+5 \rho / 2 & -4 \rho / 3 & \rho / 12 & 0 & 0 \\
0 & \rho / 12 & -4 \rho / 3 & 1+5 \rho / 2 & -4 \rho / 3 & \rho / 12 & 0 \\
0 & 0 & \rho / 12 & -4 \rho / 3 & 1+5 \rho / 2 & -4 \rho / 3 & \rho / 12 \\
0 & 0 & 0 & 0 & 0 & 1 & 0 \\
0 & 0 & 0 & 0 & 0 & 0 & 1
\end{array}\right)
$$

and

$$
B=\left(\begin{array}{ccccccc}
1 & 0 & 0 & 0 & 0 & 0 & 0 \\
0 & 1 & 0 & 0 & 0 & 0 & 0 \\
-\rho / 12 & 4 \rho / 3 & 1-5 \rho / 2 & 4 \rho / 3 & -\rho / 12 & 0 & 0 \\
0 & -\rho / 12 & 4 \rho / 3 & 1-5 \rho / 2 & 4 \rho / 3 & -\rho / 12 & 0 \\
0 & 0 & -\rho / 12 & 4 \rho / 3 & 1-5 \rho / 2 & 4 \rho / 3 & -\rho / 12 \\
0 & 0 & 0 & 0 & 0 & 1 & 0 \\
0 & 0 & 0 & 0 & 0 & 0 & 1
\end{array}\right)
$$

\section{$6 \quad$ Numerical Results}

The diffusion problem (5) is solved by using 7 different numerical methods, in order to compare their solutions with each other and with the exact analytical solution of the problem.

To solve the problem (5) we use a $20 \times 20$ grid in the $(x, t)$-field to apply several methods. All of them gave satisfactory results, except the forward Euler method and Heuns method where the numerical solution was instable. The maximum relative error at the gridpoints was of order $10^{-7}$ for the conventional CrankNicoloson method, the backward Euler method, the BDF2, BDF3. This error was reduced when using the method (22), as illustrated in Fig. 1 and Fig. 2 where the exact solution (6) and numerical solution is presented at the time $t_{\text {end }}=0.01$ when using respectively the conventional Crank-Nicolson method and method (22). In both cases the problem was solved under the conditions $H_{0}=5, H_{c}=100, B_{c}=1.6$,

\begin{tabular}{|c|c|c|c|c|c|c|c|}
\hline & $\mathrm{CN} 2$ & CN4 & Heun & BDF2 & BDF3 & $\begin{array}{c}\text { forward } \\
\text { Euler }\end{array}$ & $\begin{array}{l}\text { backward } \\
\text { Euler }\end{array}$ \\
\hline $\begin{array}{l}n_{x}=20 \\
n_{t}=20\end{array}$ & 0.02590 & 0.01094 & instable & 0.02585 & 0.02554 & instable & 0.02508 \\
\hline $\begin{array}{l}n_{x}=40 \\
n_{t}=40\end{array}$ & 0.02640 & 0.01095 & instable & 0.02638 & 0.02583 & instable & 0.02231 \\
\hline $\begin{array}{l}n_{x}=80 \\
n_{t}=80\end{array}$ & 0.02666 & 0.01703 & instable & 0.02665 & 0.02819 & instable & 0.02352 \\
\hline
\end{tabular}
$\sigma=3 \times 10^{6}, n=7$ and a $\left(n_{x} \times n_{t}\right)$-grid in the $(x, t)$ plane.

Table 1: Average errors at $t=t_{\text {end }}$ for problem (5) for different time stepping methods

In Table 1 a survey is presented of the average error

$$
e_{\text {avg }}=\frac{1}{n_{x}} \sum_{i=1}^{n_{x}}\left|H\left(x_{i}, t_{\text {end }}\right)-H_{e x}\left(x_{i}, t_{\text {end }}\right)\right| / H_{e x}\left(x_{i}, t_{\text {end }}\right)
$$

for several numerical methods where CN2 is the notation for the conventional Crank Nicolson method (21) and CN4 for its improved version (22). The exact solution $H_{e x}$ can be deduced from (6) and (4).

The steep increase of $B$ from $-B_{c}$ to $B_{c}$ after different time periods $(t=0.01, t=0.05, t=0.1)$ is shown in Figure 3, where the wave grows in the positive direction of the $x$ - and $B$-axis as $t$ increases (thickness of lines grows as $t$ increases).

\section{Lamination model}

As the validity of method (22) is proven on the testexample (5) with known exact solution, we apply the method (22) on a problem with great practical relevance as described below. The lamination model can 


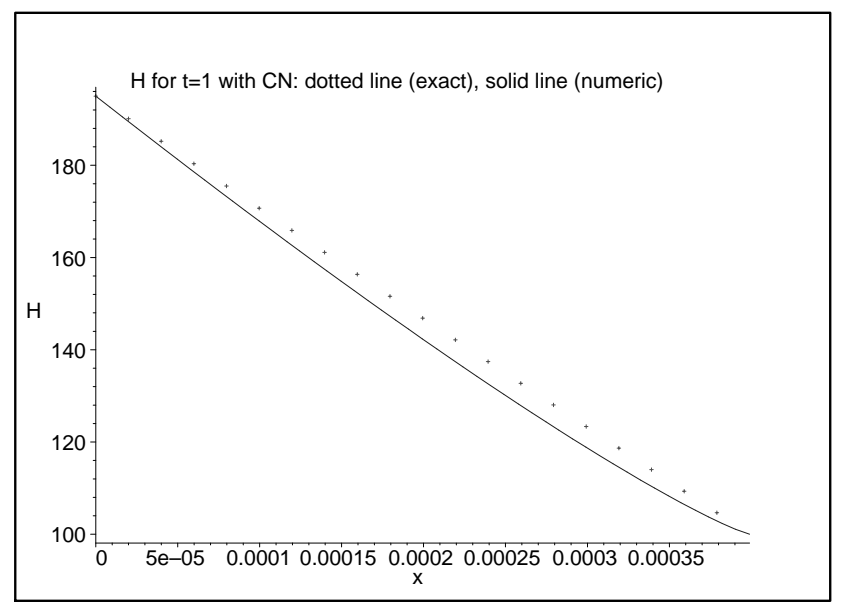

Figure 1: Exact and numerical solution of problem (5) throughout the lamination with conventional Crank-Nicolson

deal with hysteretic magnetic properties of any magnetic material. These properties can be experimentally determined by using a single sheet tester or Epstein frame.

The equation to solve is the parabolic differential equation found from (1) and (2) when inserting the differential permeability $\mu_{d}=\partial B / \partial H$ :

$$
\frac{1}{\sigma} \frac{\partial^{2} H}{\partial x^{2}}=\mu_{d} \frac{\partial H}{\partial t}
$$

with boundary conditions

$$
\frac{\partial H}{\partial x}(x=d, t)=0, \quad H(x=0, t)=H_{0} \sin (\lambda 2 \pi t)
$$

and initial conditions

$$
H(x, t=0)=0 .
$$

where $2 d$ is the thickness of the lamination.

Figure 5 shows the hysteresis loops produced by method (22) of problem (28)-(30) for $H_{0}=1500$ and parameters as in Table 2. The magnetic properties used, are these of an electrical silicon steel M700-50. A distinction is made between static and dynamic loops based on respectively surface fields and average inductions in [3]. For small values of $\sigma$ the variation in the $B$-field is limited throughout the lamination, which is depicted in Fig. 5.6 by the resemblance of the static and the dynamic loop. But comparing Fig. 5.3 and Fig. 5.7 learns that the effect of a smaller $\sigma$ is neutralized by higher differential permeability, which can also be seen from (28. As for augmented frequencies $\lambda$, augmented thickness of the lamination $d$ or augmented permeability $\mu$, the $B$-field is very much concentrated at the border of the lamination, the peak induction of the dynamic loop is small as is illustrated by Fig. 5.4, 5.5, 5.8, 5.9, 5.10.

The situations of Fig. 5.1, 5.2 and 5.3 differ by the number of layers in the lamination. To study the evolution of the magnetic field as a function of the number of layers in the lamination, we consider the surface enclosed by the loop of the average $B$-field in the $(H, B)$-diagram with $H_{0}=1500$. The surface of the dynamic loop is the energy loss per cycle, in $J / \mathrm{m}^{3}$. Fig. 4 shows for several values of $\lambda$ that a minimum number of gridpoints along the lamination is required to avoid a reduction of this surface. In case of lower frequencies, less layers are required to reach convergence. 


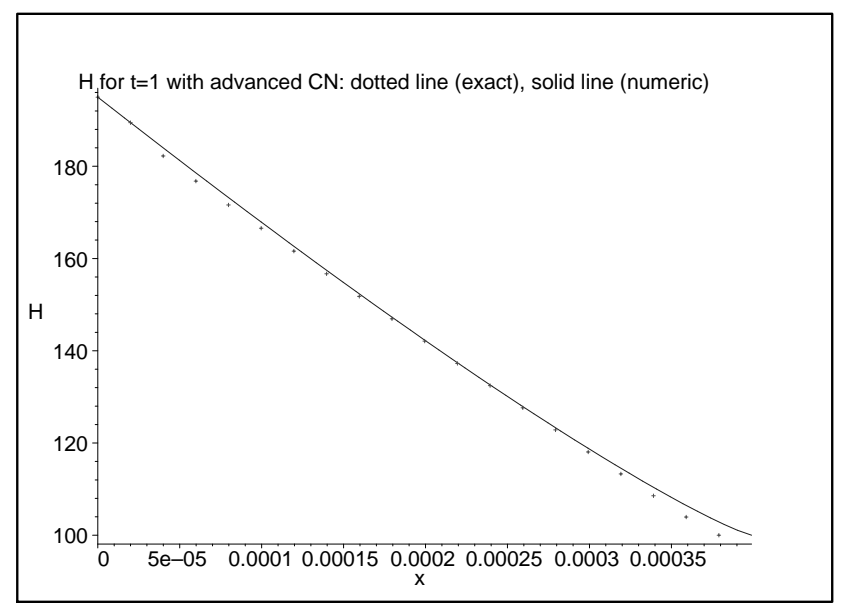

Figure 2: Exact and numerical solution of problem (5) throughout the lamination with method (22)

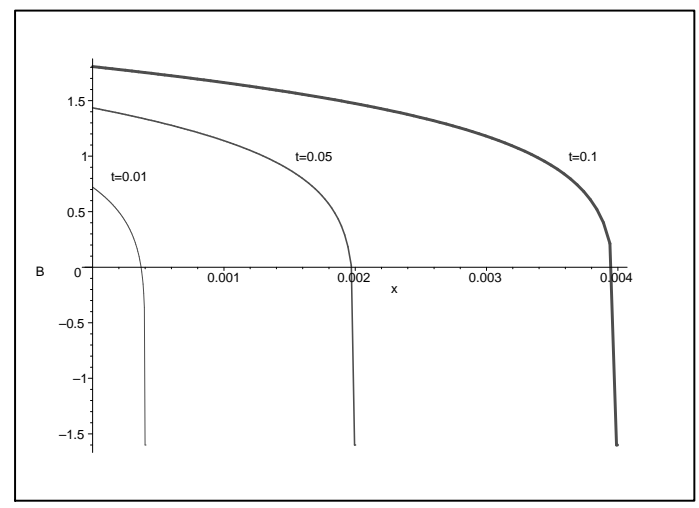

Figure 3: $B(x, t)$ as a function of $x$ for $t=0.01, t=0.05$ and $t=0.1$

\begin{tabular}{c|ccccc} 
& $n_{x}$ & $\lambda$ & $d$ & $\sigma$ & $\mu$ \\
\hline Fig. 5.1 & 5 & 1000 & 0.00025 & $3 \mathrm{e} 6$ & $\mu_{d}$ \\
Fig. 5.2 & 15 & 1000 & 0.00025 & $3 \mathrm{e} 6$ & $\mu_{d}$ \\
Fig. 5.3 & 30 & 1000 & 0.00025 & $3 \mathrm{e} 6$ & $\mu_{d}$ \\
Fig. 5.4 & 40 & 100000 & 0.00025 & $3 \mathrm{e} 6$ & $\mu_{d}$ \\
Fig. 5.5 & 40 & 100000 & 0.0025 & $3 \mathrm{e} 6$ & $\mu_{d}$ \\
Fig. 5.6 & 40 & 1000 & 0.00025 & $3 \mathrm{e} 4$ & $\mu_{d}$ \\
Fig. 5.7 & 40 & 100000 & 0.00025 & $3 \mathrm{e} 4$ & $\mu_{d}$ \\
Fig. 5.8 & 40 & 1000 & 0.00025 & $3 \mathrm{e} 6$ & $100 \mu_{d}$ \\
Fig. 5.9 & 40 & 100000 & 0.00025 & $3 \mathrm{e} 6$ & $100 \mu_{d}$ \\
Fig. 5.10 & 40 & 100000 & 0.00025 & $3 \mathrm{e} 4$ & $100 \mu_{d}$
\end{tabular}

Table 2: Parameter values for Figure 5 


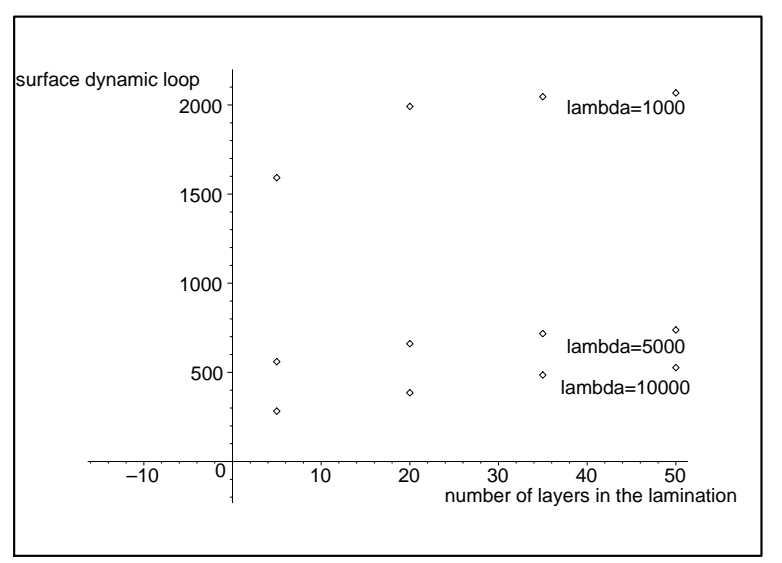

Figure 4: The surface within the dynamic hysteresis loops for the lamination model with method (22) for several frequencies as a function of $n_{x}$

\section{Conclusions}

The simulation of the nonlinear diffusion of electromagnetic fields in conducting media with gradual magnetic transitions was possible with several numerical methods. A-stability turned out to be a necessary condition to obtain accurate results. This property is one of the characteristics of the improved CrankNicolson method, which was deduced in this paper. Numerical results confirm the theoretical findings. For a theoretical testexample a reduction of the error was noticed with the improved Crank-Nicolson method, which can be explained by the higher order approximation of the derivation in space that is used with this method.

\section{References}

[1] L. Dupre, O. Bottauscio, M. Chiampi, M. Repetto, and J. Melkebeek. Modeling of Electromagnetic Phenomena in Soft Magnetic Materials Under Unidirectional Time Periodic Flux Excitations, IEEE Transactions on Magnetics 35(5): 4171 - 4184 (1999).

[2] I.D. Mayergoyz, Nonlinear Diffusion of Electromagnetic Fields, Academic Press, London, 1998.

[3] D. Philips, L. Dupre, J. Cnops and J. Melkebeek. The application of the Preisach model in magnetodynamics: theoretical and practical aspects, Journal of Magnetism and Magnetic Materials 133: $540-543(1994)$.

[4] W.E. Schiesser, The Numerical Method of Lines, Academic Press, San Diego, 1991.

[5] J. Crank, P. Nicolson. A practical method for numerical evaluation of solutions of partial differential equations of the heat conduction type, Proc. Camb. Phil. Soc. 43: 50 - 67 (1947).

[6] E. Hairer, S. Norsett and G. Wanner, Solving ordinary differential equations I: Nonstiff problems, second edition, Springer Verlag, Berlin, 1993.

[7] W.E. Milne. Numerical integration of ordinary differential equations, American Mathematical Monthly 33(9): 455 - 460 (1926).

[8] J.C. Butcher, Numerical Methods for Ordinary Differential Equations, John Wiley, 2003.

[9] P. Wesseling. von Neumann stability conditions for the convection-diffusion eqation, IMA Journal of Numerical Analysis 16(4): 583 - 598 (1996). 

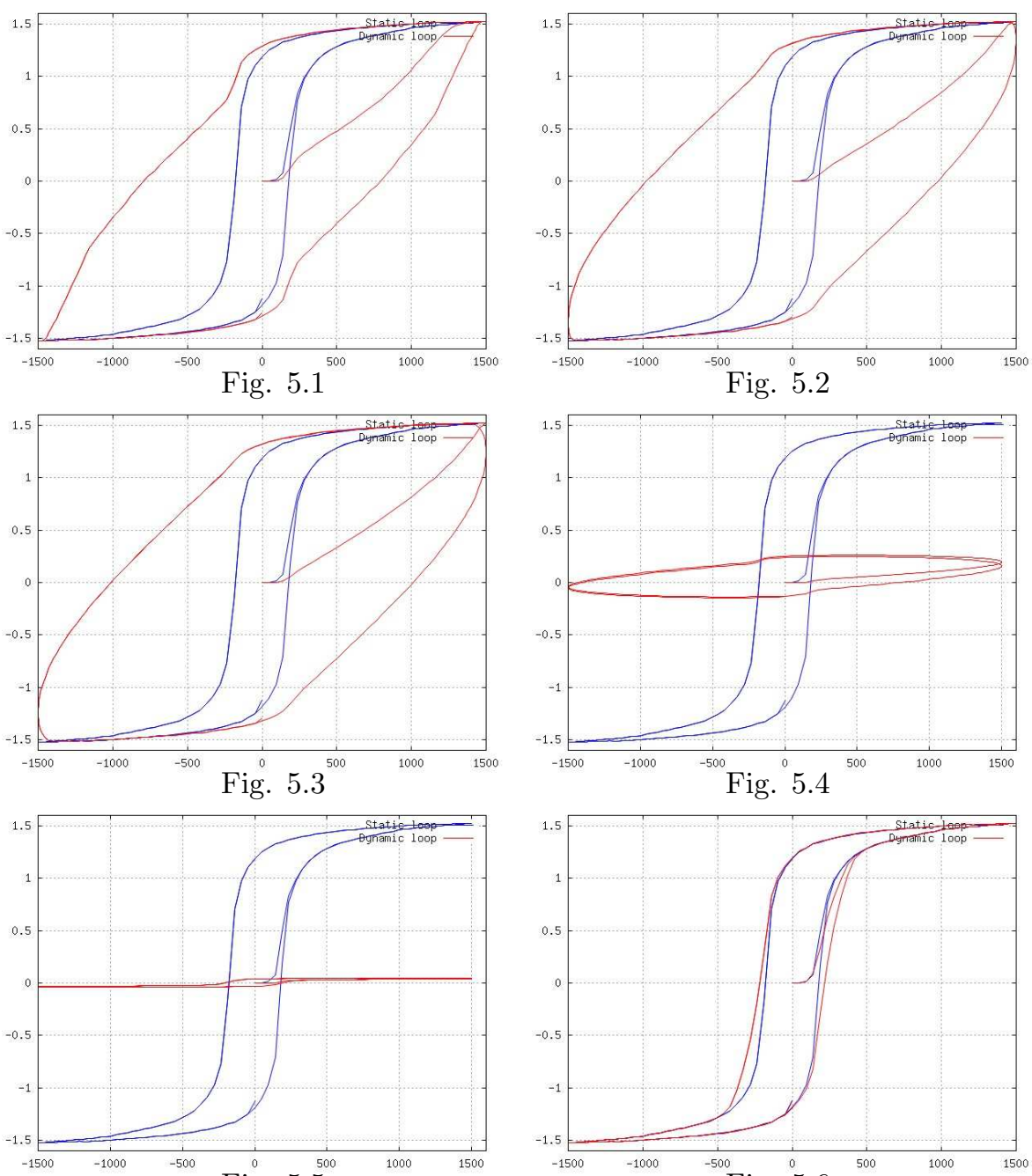

Fig. 5.5
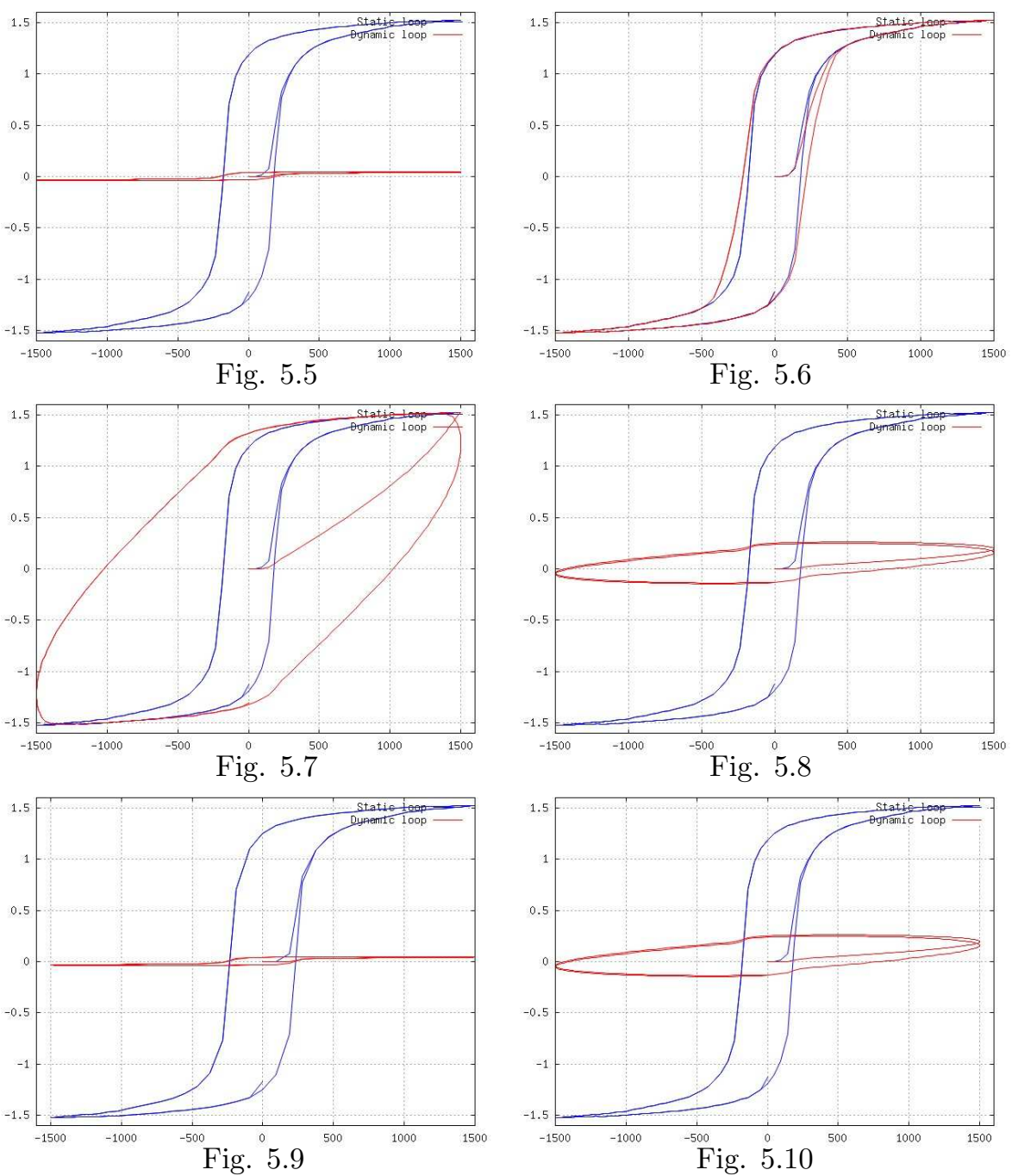

Figure 5: Hysteresis loops (static and dynamic) for the lamination model with method (22) and parameters as in Table 2 